\title{
L'Exemple d'Une Dramaturgie Prospective et Documentaire Pour Ça ira (1) Fin de Louis de Joël Pommerat
}

\author{
Marion Boudier \\ Université de Picardie Jules Verne, Amiens, France \\ E-mail: marion.boudier@u-picardie.fr
}

\section{Resumé}

Cet article propose une description et une théorisation du travail dramaturgique réalisé par Marion Boudier aux côtés de l'auteur-metteur en scène Joël Pommerat pour la création de Ça ira (1) Fin de Louis (créé en 2015 et présenté à la Mostra Internacional de Teatro de São Paulo en 2016). Comment travailler à partir des archives de la Révolution française pour accompagner un auteur-metteur en scène qui écrit ses textes avec la scène pendant les répétitions ? Marion Boudier présente ce qu'elle nomme une " dramaturgie prospective et documentaire ». Cet accompagnement de l'écriture se distingue des pratiques classiques de la dramaturgie. En effet, pour entrer en écriture, Joël Pommerat a dirigé ses comédiens dans des improvisations longuement préparées et nourries par une importante documentation historique : plutôt que d'éclairer un texte préalable en vue de son passage à la scène, le travail dramaturgique consiste alors, dans ce cas, à proposer une matière documentaire pertinente pour le sujet choisi et potentiellement riche pour le théâtre, puis à réagir à ses évolutions scéniques. Ainsi cette dramaturgie est une sorte d'enquête, menée au service de l'auteur-metteur en scène et de ses comédiens tout au long du processus de création.

Dramaturgie. Prospection. Documentation (archive). Écriture de plateau. Révolution Française.
Abstract

This article proposes a description and theorization of the dramaturgical work done by Marion Boudier alongside author-director Joël Pommerat for the creation of Ça ira (1) Fin de Louis (created in 2015 and represented at São Paulo International Theater Exhibition in 2016). How to work from the archives of the French Revolution to accompany an author-director who writes his texts with the scene during rehearsals? Marion Boudier presents what she calls "prospective and documentary dramaturgy". This accompaniment of scripture is distinguished from classical dramaturgy practices. In order to enter scripture, Joël Pommerat directed his actors in long-prepared and nourished improvisations for an important historical documentation. Rather than clarifying a previous text with the purpose of transposing it into the scene, the dramaturgical work then consists in proposing a documentary material relevant to the chosen theme and potentially resourceful for the theater, then reacting to its scenic evolutions. This way, the dramaturgy is a kind of investigation, carried out at the service of the author-director and his actors throughout the process of creation. Keywords

Dramaturgy. Prospection. Documentation (archive). Stage writing. French Revolution. 
J'accompagne Joël Pommerat depuis 2013 en tant que collaboratrice à la dramaturgie pour des projets au théâtre et à l'opéra. Cet article propose une description et une théorisation du travail de dramaturge à travers l'exemple de Ça ira (1) Fin de Louis créé à Mons - Capitale de la Culture en 2015. Cette contribution s'inspire d'une conférence donnée lors de la Biennale des écritures du réel à Marseille en avril 2018. Elle synthétise certaines analyses développées plus amplement dans l'ouvrage Avec Joël Pommerat, tome 2, paru aux éditions Actes Sud et lauréat du Prix du Syndicat de la critique - meilleur livre sur le théâtre - en 2019.

Ça ira (1) Fin de Louis de Joël Pommerat est une fiction politique contemporaine qui retrace les débuts de la Révolution française, depuis la crise économique et la tentative de réforme fiscale en 1786-1787 jusqu'à la montée de la contre-révolution dans les années 1790-91. Cette archéologie du régime démocratique est porté par quatorze comédiens qui, pendant $4 \mathrm{~h} 30$ de spectacle, incarnent plus de soixante personnages, principalement des orateurs politiques, membres de l'entourage royal, députés aux Etats généraux puis à l'Assemblée nationale, parisiens réunis en assemblées de quartier. En immergeant le public dans ces débats à travers une scénographie englobante (la salle devient l'Assemblée), Ça ira (1) Fin de Louis propose une histoire vivante, révélatrice de nombreuses filiations entre hier et aujourd'hui et porteuse d'enjeux universels tels la liberté, le rapport à la violence, la représentation politique... Les spectateurs brésiliens qui ont vu le spectacle à Sao Paulo au moment de la destitution de la présidente Dilma Roussef ont sans doute pu y retrouver des propos ou des situations politiques et humaines qui leur étaient proches. Dans chaque ville et chaque pays où Ça ira (1) Fin de Louis a été présenté, des échos se sont ainsi produits entre les événements révolutionnaires et l'actualité ou le contexte. Pourtant c'est bien de 1789 dont il s'agit : comment Joël Pommerat réactive-t-il ce mythe ? Quelle démarche d'écriture inventer pour mettre les spectateurs « au présent du passé »? Je ne suis pas historienne mais dramaturge, en quoi mon travail a-t-il consisté au sein de cette création?

La dramaturgie est un terme polysémique qui sert à désigner deux pratiques distinctes : la composition des pièces de théâtre (le travail de l'auteur) et la pensée de leur passage à la scène (travail du Dramaturg en allemand ou du dramaturgista en espagnol - le français ne possède qu'un seul terme, source de confusion). Joël Pommerat a la particularité d'écrire ses spectacles en lien étroit avec la scène, en même temps qu'il les répète et les met en scène avec ses comédiens. Dans cette manière de créer, l'écriture et la mise en scène s'inventent dans le même temps, pendant les répétitions. N'allant plus du texte vers la scène mais de la scène vers le texte, c'est la démarche créative qui fait l'œuvre. (BOUDIER, 2015) Ce processus d'écriture avec la scène déplace le rôle du dramaturge. Avec un « écrivain de plateau » (TACKELS, 2016) tel Joël Pommerat, l'activité théâtrale cesse d'être un art à " deux temps » (GOUHIER, 1989) (l'écriture d'un texte puis la mise en scène de ce texte), deux temps qui permettent, dans la plupart des cas, de distinguer dramaturge-auteur (Dramatiker) et dramaturge-maïeuticien (Dramaturg). Dès lors que le texte est écrit en même temps qu'est élaborée sa mise en scène, la « composition » et la «pensée du passage à la scène » forment un même mouvement. Qu'implique ce double processus d'écriture-mise en scène pour le travail de dramaturge ? En quoi « dramaturger ${ }^{1}$ » avec Joël Pommerat est-il di-

$1 \mathrm{Ce}$ verbe est un néologisme. Le terme «dramaturgiste » parfois utilisé au Québec n'est pas encore répandu en France. 
fférent d'autres pratiques de la dramaturgie?

\section{La « dramaturgie prospective »}

Le plus souvent, la dramaturgie désigne un travail d'interprétation du texte en amont des répétitions (dossier dramaturgique préalable, travail à la table), herméneutique qui se prolonge dans certains cas par un accompagnement à la mise en scène (dramaturgie de plateau, regard extérieur). La dramaturgie consiste alors à inventer un lien entre un texte et sa représentation, à motiver et à éclairer des choix pour la scène ${ }^{2}$. On peut repérer des fonctions récurrentes, cumulées ou non, qui font surtout du dramaturge un maïeuticien : lecteur qui explicite le contexte, la structure et les effets de sens possibles du texte, documentaliste qui réunit autour du texte ou du sujet un ensemble de sources qu'il propose aux membres de l'équipe, parfois adaptateur ou traducteur, premier spectateur qui observe la répétition et la commente afin de mettre en évidence ses orientations.

Avant même «l'invention » de la mise en scène au $\mathrm{XIX}^{\mathrm{e}}$ siècle, cette fonction a été initiée par Gotthold Ephraim Lessing à la fin du XVIII siècle au Théâtre national de Hambourg à travers sa revue Dramaturgie de Hambourg : « revue critique » dédiée au théâtre et chargée d'« accompagner chaque pas qu'y fera l'art du poète comme celui du comédien ». (LESSING, 2010) En réalité, il me semble que ce processus d'accompagnement est transversal et partagé : tous les membres d'une équipe de création font, d'une manière ou d'une autre et plus ou moins consciemment, de la dramaturgie dès lors qu'ils s'interrogent sur leurs choix esthétiques, sur le sens à mettre en jeu et ses représentations possibles. La dramaturgie est,

2 Voir notamment Joseph Danan, Qu'est-ce que la dramaturgie? , Arles, Actes Sud-Papiers, collection "Apprendre", 2010 ; Anne-Françoise Benhamou, Dramaturgies de plateau, Les Solitaires comme l'écrivait Bernard Dort, « un état d'esprit », une prise de conscience et une pratique du choix responsable : " une pratique transversale. Non une activité en soi ». (DORT, 1986) De manière radicale, on pourrait donc avancer qu'il existe autant de définitions et de pratiques de la dramaturgie que d'équipes et de processus de création : le mode opératoire est à réinventer à chaque projet.

Qu'il soit auteur et metteur en scène de ses textes et puisse être son propre dramaturge (Dramaturg) n'empêche pas que Joël Pommerat ressente le besoin d'être accompagné dans sa démarche. Même si elle n'est plus à « deux temps ", la création n'abolit pas totalement la double nature du théâtre (texte et scène). Elle continue de se développer selon des moments et des étapes avec des accents spécifiques, plus ou moins textuels ou scéniques, collectifs ou solitaires. Pour Ça ira (1) Fin de Louis, j'ai accompagné certains de ces moments, depuis la recherche d'une thématique pour le spectacle, le développement de l'écriture lors des répétitions avec les comédiens et collaborateurs artistiques, jusqu'à l'édition de la pièce. Joël Pommerat développant son écriture pendant les répétitions, notamment à travers des improvisations dirigées, mon accompagnement concerne également les comédiens. Pour diriger ses acteurs dans des improvisations fertiles, Joël Pommerat insiste en effet sur la nécessité qu'ils soient « nourris » et que leurs imaginaires soient « musclés » grâce à une abondante documentation. Ma collaboration à la dramaturgie pour Ça ira (1) Fin de Louis est en grande partie liée à cette exigence documentaire qui est une caractéristique de la démarche créative de Joël Pommerat : la documentation est un matériau indispensable pour qu'il puisse entrer en écriture en collaboration avec ses comédiens. Le travail dramaturgique consiste alors à proposer une matière potentielle et à réagir à ses évolu- 
tions scéniques, plutôt que d'éclairer une matière préexistante en vue de son passage à la scène. Ma pratique de la dramaturgie diffère ainsi de celle du « théâtre à deux temps ». Je propose de la nommer " dramaturgie prospective » et d'identifier quatre opérations principales de son déroulement : identifier un sujet ou une thématique de création, rassembler un matériau pertinent pour le théâtre par rapport à ce sujet, réfléchir à une méthodologie de travail à partir de ce matériau, accompagner ses diverses évolutions scéniques.

\section{Comment raconter une histoire} dont on connaît déjà la fin?

Prospecter signifie d'abord chercher, défricher, préparer le terrain. Pour Ça ira (1) Fin de Louis, la réflexion avait commencé dès l'automne 2013 : avant que ne s'impose le choix d'écrire sur la Révolution française, j'ai travaillé notamment sur des hypothèses de spectacle portant sur l'économie et les grands chefs d'Etat. Joël Pommerat envisageait d'écrire une épopée, de questionner les liens entre idéologie et actions ainsi que le mythe de la liberté individuelle. De lectures en lectures s'est affirmé son désir de travailler sur la Révolution Française. Lors de cette première étape de prospection, c'est un immense plaisir de participer à l'émergence d'un projet, de voir un thème s'imposer et des idées se cristalliser jusqu'à susciter un fort désir d'écriture, une conviction (même si beaucoup de choses sont encore intuitives) à partir de laquelle va s'engager un travail de plusieurs mois avec toute une équipe.

Une fois une direction de recherche validée, la recherche dramaturgique prospective se précise de plus en plus pour accompag-

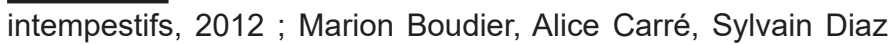
et Barbara Métais-Chastanier, De quoi la dramaturgie est-elle le nom ?, L'Harmattan, coll. “Univers théâtral”, mars 2014. ner le développement de l'écriture au jour le jour. II s'agit de réunir des textes, de l'iconographie, des films, un ensemble de matériaux qui déclencheront et accompagneront la naissance de situations et de personnages jusqu'à l'écriture de dialogues. Pour plonger au cœur de la Révolution française sans préjugés ni partis-pris, Joël Pommerait voulait travailler le plus possible à partir de paroles directes : discours, procès-verbaux d'assemblées, correspondances, mémoires. J'ai donc rassemblé des sources primaires, des idées, des discours, et non des biographies commentées ou des interprétations de l'événement. Partir des documents allait être essentiel pour " nourrir » les acteurs mais aussi pour se démarquer d'une historiographie très politisée, voire partisane, de la Révolution, que ce soit dans la tradition de gauche dans laquelle s'inscrivait le 1789 du Théâtre du Soleil d'Ariane Mnouchkine en montrant comment la révolution a été confisquée au peuple, ou dans la lecture de François Furet qui assimile la révolution à l'origine du totalitarisme. Partir des archives et des faits (chronique) était une tentative d'écrire sans préjugés ni interprétation préétablie pour commencer la création " avec innocence ", comme si nous ne connaissions pas la fin de l'histoire ni ses héros.

L'un des premiers grands principes de travail a donc été de partir le plus possible de la connaissance des faits en lisant différentes chroniques de la Révolution au jour le jour, de partir de la lecture de témoignages directs, notamment des procès verbaux d'assemblée ou des correspondances, de travailler sur les idées des révolutionnaires et non à partir de leurs biographies. Plutôt que de raconter l'histoire des grands hommes et celle des grands événements. Joël Pommerat souhaitait proposer une histoire à hauteur d'homme, faite en partie par des anonymes (« à l'époque, Robespierre c'est Monsieur Dupont » 
écrit-il dans une note). Hormis Yvain Juillard, choisi pour incarner le roi, les acteurs ont tous exploré différents positionnements sociaux et idéologiques avant d'approfondir la construction de leur(s) personnage(s). Ces personnages ne sont pas des décalques de personnages historiques réels, mais le résultat d'une écriture inspirée par de multiples archives et par les comédiens eux-mêmes. La députée Lefranc, par exemple n'est pas Robespierre ni Marat, mais une invention à partir des propos de Robespierre, de Marat, Desmoulins, Lanjuinais et d'autres encore, incorporés par Saadia Bentaïeb et chargés de ses propres émotions et mémoires intimes.

Un autre postulat de départ était de faire comme si on ne connaissait pas la fin : c'était nous astreindre à chercher le " présent du passé ", à retrouver le surgissement, le bouillonnement, l'incertitude, l'imprévisibilité propre au réel révolutionnaire. Si la réalité est ce qui est perçue et interprétée, le réel est précisément ce qui nous échappe, «ce qui ne finit pas de ne pas s'écrire » (Lacan), ce qui nous surprend, ce qui nous comprend plus qu'on ne le comprend. II s'agissait donc de penser à nouveau cette histoire comme un problème, une énigme, pour la redécouvrir plutôt que de dérouler le récit établi des grands moments d'une histoire patrimonialisée / matrimonialisée.

Avant le début des répétitions avec l'équipe de création, deux ateliers d'exploration ouverts à des comédiens extérieurs à la Compagnie ont permis de préciser ces premières pistes en se confrontant aux archives et à diverses manières de les théâtraliser. La réflexion dramaturgique a donc servi à élaborer des hypothèses méthodologiques plutôt qu'à orienter une « lecture » de la Révolution Française. Même si le travail de collecte de documents et cette réflexion méthodologique commence avant le début des répétitions, il ne s'agit pas d'une dramaturgie préalable ${ }^{3}$ au sens d'une hypothèse narrative et interprétative que la mise en scène viendrait ensuite expérimenter ou concrétiser. II s'agit d'une enquête. La démarche a été processuelle, tâtonnante. L'état d'esprit est celui de la recherche, un questionnement permanent à partir d'hypothèses, d'essais et d'expérimentations.

\section{Faire parler les archives}

\section{L'historien Guillaume Mazeau ${ }^{4}$ a été un} collaborateur essentiel pour la recherche d'archives nécessaire à l'écriture de plateau. Je l'ai d'abord sollicité comme « expert » pensant qu'il pourrait donner quelques cours ou conférence aux comédiens, mais finalement, il a accompagné l'ensemble de la création et nous avons travaillé en binôme, ce qui constitue une singularité du processus de création de Ça ira (1) Fin de Louis. Assistés de plusieurs stagiaires, nous formions « l'équipe dramaturgie » dont j'organisais le travail.

A partir des notes de Joël Pommerat, dont la forme et le contenu évoluent en permanence, nous avons d'abord travaillé de manière quantitative en accumulant des sources (plusieurs récits d'un même événement, plusieurs discours défendant la même position ou un fragment entier des archives parlementaires par exemple). Tentative d'épuisement d'une matière inépuisable... A mesure que l'écriture a progressé, lorsque des grandes séquences historiques ont été identifiées pour devenir des scènes,

\footnotetext{
3 Voir « Préalable » et « Dramaturgie de concept » dans Marion Boudier, Alice Carré, Sylvain Diaz et Barbara Métais-Chastanier, De quoi la dramaturgie est-elle le nom ?, L'Harmattan, coll. Univers théâtral, 2014.

4 Guillaume Mazeau est Maître de conférences à l'Institut d'Histoire de la Révolution Française, Université Paris 1 - Panthéon Sorbonne. Il est notamment l'auteur du Bain de l'histoire (Champ Vallon, 2009) et de Pour quoi faire la Révolution (Agone, 2012, en collaboration avec Jean-Luc Chappey, Bernard Gainot, Frédéric Régent et Pierre Serna).
} 
la recherche documentaire s'est resserrée pour devenir plus qualitative. Pour chaque moments historiques de ces années 17861791 et parfois aussi pour des idéologies politiques, nous avons constitué des dossiers documentaires, regroupant de larges extraits des Archives parlementaires, des correspondances de députés, des extraits de presse, des journaux intimes, manifeste, pétition et autres prises de position orales ou écrites. Nous avons principalement sélectionné des documents historiques dans lesquels s'exprimaient des points de vue singuliers, qu'ils appartiennent au genre du discours, du témoignage ou du récit.

Une pensée du passage à la scène guidait en permanence cette sélection documentaire : il ne s'agit pas uniquement de sélectionner un matériau historiquement pertinent, mais un matériau plus susceptible qu'un autre de devenir théâtre, un événement qui pourrait faire une bonne situation dramatique, un texte qui aidera l'acteur à trouver un état, des extraits d'une correspondance à réécrire pour en extraire un point de vue singulier... En plus des discours, j'ai par exemple rassemblé une série de détails et d'anecdotes très concrètes sur des problèmes d'organisation de la parole à l'Assemblée, sur la chaleur, les odeurs, sur les attitudes de certains députés... Cette dramaturgie est proche du travail d'un documentaliste mais s'en distingue en raison de sa grande proximité avec le travail scénique. Elle a lieu en interaction avec le plateau : c'est pour cela que j'emploie également l'expression « documentation au plateau » pour décrire mon travail. Le matériau dramaturgique proposé influence la direction que peut prendre une scène et réciproquement, l'avancée de l'écriture réoriente en permanence la recherche documentaire.

\section{Une dramaturgie au service des} comédien·ne.s

La collecte des archives est une première étape avant ce qu'on pourrait appeler le « traitement dramaturgique » du matériau pour désigner les opérations d'appropriation et de transformation qui conduisent du document à la parole théâtrale. Si certaines improvisations des comédiens ont pu être menées directement à partir de sources «brutes », d'autres archives avaient subi une première transformation : surlignage, sélection, découpage, montage, réécriture, reformulation, mise en perspective... Guillaume Mazeau et moi avons par exemple réécrit certaines sources narratives sous la forme de brèves prises de parole pour en faciliter l'accès aux comédien.ne-s. Nous avons dressé des listes d'éléments de langage tirés de ces archives. II nous est également arrivé de «fabriquer des sources » en fusionnant des textes ou en recontextualisant certains arguments (et dans ce cas la présence de l'historien était essentielle pour ne pas trahir l'histoire) : plusieurs discours du député conservateur du tiers état Malouet synthétisés en une page, par exemple, ou des arguments tenus en 1793 contre la violence utilisés pour travailler une scène sur le même thème mais située en 1789 .

Les dossiers documentaires contiennent aussi des documents appartenants à d'autres époques historique. Par exemple, dans un dossier consacré à la violence révolutionnaire : les textes de Marat sur les émeutes populaires, Robespierre sur le droit à l'insurrection, la pétition de Pauline Léon pour l'armement des femmes et des textes plus contemporains, comme une sélection de Chroniques de la révolution égyptienne d'El Aswany pour leur appel à l'engagement et la description de crimes policiers, des textes de la Fraction Armée Rouge sur la guérilla 
urbaine... Nous avons également échangé des liens Youtube montrant des manifestations en Égypte et en Tunisie, la fraternisation de la police avec des manifestants, des images de l'INA sur les chars soviétiques à Prague en 1968... Ces anachronismes contrôlés fonctionnent comme des analogies dans le but d'aider les comédiens à entrer dans l'émotion et des états extrêmes.

La transmission des documents aux comédien'ne·s, à travers l'organisation des dossiers documentaires, des lectures collectives à la table ou des entretiens individuels, est un moment essentiel pour que l'archive puisse devenir un support d'imagination et un véritable combustible pour l'écriture. Sans l'archive, les comédiens butaient sur des approximations ou des clichés. Inversement, sans leurs imaginaires et leurs corps qui permettaient de figurer le passé en lui redonnant l'effectivité d'un temps présent vécu, l'archive serait restée muette. Dans la démarche de Joël Pommerat, l'improvisation dirigée permet ce processus d'appropriation, d'interprétation et de compréhension des archives. Deux grands axes méthodologiques se sont peu à peu dégagés pour les improvisations : un travail individuel de production de discours politique, la mise en scène collective de situations historiques. Avec les discours individuels, nous cherchions à identifier les critères d'une («vraie ») parole politique, la moins fabriquée possible, pour éviter de voir les comédien.ne.s reproduire des postures politiques. II s'agissait de leur donner des textes pour les « charger » et de les «muscler politiquement » avant les improvisations collectives dirigées. Ces improvisations solitaires de discours consistaient pour les comédien'ne-s à s'approprier le contenu d'une archive et à le reformuler. II s'agissait de mémoriser un contenu, des arguments, et non un texte dans son ensemble, afin d'être capable d'improviser à partir de lui. Ce faisant, les comédien.ne-s pouvaient prendre de la liberté par rapport au registre de l'hypotexte, traduire, moderniser la langue d'époque. En accumulant plusieurs discours d'une même tonalité et couleur politiques, on faisait petit à petit émerger un personnage : par exemple, nous avions été particulièrement frappé par un discours de Barthès sur « la supériorité de la race noble " réimprovisé par le jeune Simon Verjans. Nous lui avons ensuite donné plusieurs discours réactionnaires à travailler : Burke, Seinac de Meilhan, et même des contemporains comme Mauras ou Finkelkraut. Ainsi a été ainsi construit le personnage du député de la noblesse De Lacanaux, contre-révolutionnaire très actif lors des dernières négociations pour éviter que le tiers ne se déclare Assemblée Nationale ou lors de l'arrivée de femmes de Paris à Versailles. Le trajet de l'écriture part donc des discours pour aller vers le personnage en passant par le comédien et tout ce qu'il apporte en réimprovisant la source. Ce travail sur l'improvisation de discours solitaire n'a pas toujours abouti à une silhouette ou à une identité, mais il a permis de préciser des positionnements idéologiques et émotionnels possibles, de dégager des thèmes et des arguments, d'imaginer des débats ou des situations.

Pour les improvisations collective dirigées, Joël Pommerat indiquait par exemple un moment ou une date, un lieu, des thèmes à aborder (le 13 juillet au soir, dans une réunion de quartier à Paris, la violence, la peur, la faim...) et il distribuait les comédiens ou leur proposait de choisir un positionnement (l'homme politisé modéré, une femme préoccupée par sa survie, un homme politisé radical partisan de la violence armée, un indécis...). De cette manière, sans le savoir nous avons parfois fait de l'histoire contrefactuelle : à la fin de certaines improvisation située le 14 juillet, la Bastille n'était pas pri- 
se ! L'improvisation comme exploration des possibles d'une situation historique nous a permis de mieux comprendre les interactions entre les différentes forces en présence et de ressentir la surprise ou l'urgence de la prise de décision dans ce contexte. A travers l'improvisation, les documents du passé cessaient de n'être que du passé pour redevenir du « futur inaccompli $»^{5}$.

Reprise et approfondie pendant plusieurs jours, l'improvisation permettait de dégager un premier canevas ou synopsis des scènes ainsi que des bribes de dialogues à partir desquelles Joël Pommerat écrivait. Joël Pommerat préfère écrire à partir de ce qu'il a observé et ressenti sur la scène : il met en forme certains éléments ayant eu lieu au plateau ou propose tout autre chose ; il ne s'agit pas de la sténographie de la parole improvisée. L'écriture se déploie à travers une dynamique de palimpsestes successifs à partir des documents historiques à travers plusieurs étapes collective de pré-écriture («traitement dramaturgique » du matériau, improvisations des comédiens) puis de réécriture en solitaire par Joël Pommerat. Dans la dynamique de création collaborative qui est celle de la Compagnie Louis Brouillard, le dramaturge n'a aucune autorité quant à la question du savoir et du texte ; chacun'e à sa manière y contribue, par le jeu, par les costumes, par la documentation, etc., à partir des indications données par Joël Pommerat qui fixe et signe le spectacle au terme du processus.

Joël Pommerat souhaitait écrire une «fiction vraie » à partir d'événements historiques, en tant que dramaturge, je me suis donnée comme tâche d'être une sorte de vigie de cet oxymore : fournir du vrai, du réel, des faits historiques, des archives, sans jamais perdre de vue que nous faisions du théâtre, une vérité fabriquée, non scientifi-

5 Voir Paul Ricœur, Temps et récit 3. Le temps raconté, Paris, Seuil, coll. Point, p. 329-348, 1991 (1985). que mais humaine et sensible. Je conçois la dramaturgie prospective et documentaire avant tout comme un outil pratique, inspirant et enrichissant qui, tout en étant précis et le plus exhaustif possible, se doit de trouver une efficience scénique, de produire du jeu, des états émotionnels, des expériences de pensée. "Puisse chacun être son propre historien. Alors il vivra avec plus de soin et d'exigence " comme disait Brecht... ${ }^{6}$

Dans le double contexte postmoderne de la fin des grands récits émancipateurs (J.-F. Lyotard) et du théâtre postdramatique (Lehmann), Ça ira (1) Fin de Louis ré-affirme le goût de Joël Pommerat pour le récit. Tout en déjouant les codes de l'épopée, il propose aux spectateurs une expérience : à « hauteur d'hommes », en déconstruisant le mythe et la patrimonialisation, il invite à revivre le passé comme du présent et nous rappelle à quel point la démocratie demeure un enjeu pour aujourd'hui.

\section{Références}

BENHAMOU, Anne-Françoise, Dramaturgies de plateau, Les Solitaires Intempestifs, 2012.

BOUDIER, Marion, Avec Joël Pommerat, un monde complexe, Actes Sud-Papiers, coll. "Apprendre", 2015.

BOUDIER, Marion; CARRÉ, Alice; DIAZ, Sylvain; MÉTAIS-CHASTANIER, Barbara, De quoi la dramaturgie est-elle le nom ?, L'Harmattan, collection "Univers théâtral", mars 2014.

6 Jean-Luc Godard citant Bertolt Brecht à la fin de Tout va bien, 1972. 
DANAN, Joseph, Qu'est-ce que la dramaturgie?, Actes Sud-Papiers, collection "Apprendre", 2010.

DORT, Bernard, L'État d'esprit dramaturgique, Théâtre/ Public, nº 67, 1986.

GOUHIER, Henri, Le Théâtre et les Arts à deux temps, Flammarion, 1989.

LESSING, Dramaturgie de Hambourg [1769], trad. J.-M. Valentin, Klincksieck, coll. "Germanistique", 2010.

RICCEUR, Paul, Temps et récit 3. Le temps raconté, Seuil, coll. Point, 1991 (1985).

TACKELS, BRUNO, Les Écritures de plateau. État des lieux, Les Solitaires Intempestifs, 2016.

Recebido: 02/09/2019

Aprovado: 24/11/2019 